\title{
Marichette. Lettres acadiennes 1895-1898 en polonais : stratégies de traduction
}

\author{
Alicja Żuchelkowska \\ Université Adam Mickiewicz
}

\section{Introduction}

Le projet de recherche "Le contact des marges : le Canada francophone minoritaire en Pologne» auquel nous avons participé dans les années 2006-2007 visait à frayer le chemin en vue d'élaborer un programme d'envergure sur la traduction de la littérature canadienne française en Pologne. La première étape de ce programme de recherche s'était donnée comme objectif la traduction et la diffusion de six œuvres littéraires acadiennes et l'étude de leur réception en Pologne ${ }^{1}$. Les «Lettres de Marichette » d'Émilie C. LeBlanc (dont le pseudonyme était Marichette) n'ont pas fait partie de ce projet, bien qu'elles aient une valeur indubitable dans l'histoire de la littérature acadienne. L'absence de ces lettres est simple: malgré qu'elles figurent dans l'édition critique dirigée par Pierre Gérin et Pierre Marie Gérin, elles sont écrites dans un parler traditionnel acadien $d u X \mathrm{IX}^{\mathrm{e}}$ siècle qui représentait un obstacle infranchissable pour les étudiants polonais. Or, puisque l'objectif principal du projet Canada-Pologne fut justement de faire découvrir la diversité des littératures francophones minoritaires et de rompre avec la représentation statique du Canada à l'étranger (Giguère 2006 : 12), nous avions décidé d'insérer les «Lettres » dans le programme du cursus universitaire du deuxième cycle et de proposer aux étudiants inscrits dans le séminaire de traduction littéraire ${ }^{2}$ de traduire quelques passages choisis.

L'exercice visait deux objectifs : permettre de découvrir la diversité de la langue française et de vivre l'expérience pragmatique des échanges interculturels qui sont un véritable moteur de l'évolution des sociétés. Selon Jacques Demorgon, une fondation éthique et cognitive de l'interculturel est toujours inférieure au travail interculturel réellement effectué «à partir des contacts migratoires, des projets internationaux, d'une véritable éducation européenne » (2002 : 128) qui est une des articulations de $l^{\prime}$ « éducation mondiale » : la vraie éducation interculturelle que nous sommes censée transmettre aux étudiants ne peut être uniquement théorique. Et les étudiants ne commencent à comprendre l'importance de l'interculturel que par la

\footnotetext{
${ }^{1}$ Pour de plus amples renseignements au sujet de cette expérience de traduction, nous renvoyons au rapport de recherche signé T. Tomaszkiewicz « Transfert de la littérature acadienne en Pologne : une expérience traductologique et didactique » publié dans ce numéro d'Alternative francophone ainsi qu'à l'article signé A. Klimkiewicz et D. Merkle, 2007, « The Internet and Nomadism: Literary Translation from (Acadian) French to Polish », que l'on peut lire en ligne sur le site de l'Université Concordia, Département d'études françaises : http://francais.concordia.ca/conf07/MERKLE KLIMKIEWICZ.pdf. ${ }^{2}$ Étant donné que j'étais en charge de ce séminaire, c'est moi qui ai sélectionné, après une lecture assidue, des passages à traduire.
} 
mise en pratique et le contact personnel avec l'Autre. La traduction des «Lettres » s'inscrit particulièrement bien dans ce contexte pratique.

De plus, la reconnaissance grandissante des littératures dites " périphériques » résulte en l'émergence concomitante de nouveaux espaces littéraires. La constitution du champ littéraire acadien participe pleinement à ce mouvement. Ouvrir la voie à la traduction et par la suite à l'établissement de nouveaux ponts interculturels pourrait mener à la création d'une relation de longue durée entre différentes communautés francophones du Canada et la Pologne. Les étudiants, connaissant à présent l'existence de ces littératures francophones de l'exiguïté, pourraient suivre leur formation dans le domaine des études canadiennes et écrire des travaux scientifiques portant sur le Canada francophone minoritaire. Il faut aussi mentionner le fait que leurs traductions (que nous envisageons de publier dans un recueil littéraire dont la parution est prévue pour 2009) pourraient contribuer à une meilleure connaissance de la diversité canadienne, jusque-là complètement ignorée des Polonais.

Durant l'expérience traductionnelle, dont l'objet fut l'œuvre épistolaire de Marichette, nous avons mis l'accent non seulement sur l'acte même de traduction, quoique celui-ci constitue le pivot de notre réflexion scientifique, mais aussi sur le rôle des étudiants en tant qu'agents culturels. L'expérience nous a permis de mieux comprendre les mécanismes de compréhension d'un texte étranger de même que les mécanismes de résolution des problèmes de traduction et d'échange interculturel, bref les stratégies que les étudiants utilisent pour entamer le processus du transfert $\mathrm{d}^{\prime}$ une littérature étrangère à leur culture ${ }^{3}$.

\section{Lettres de Marichette en tant que texte hybride : problèmes de traduction}

Le premier contact avec les "Lettres de Marichette » fut un choc énorme pour nos étudiants qui ne sont pas habitués, et c'est le moindre que l'on puisse dire, à travailler avec les textes littéraires écrits dans une langue française qui n'est pas le français standard comme c'est le cas des «Lettres de Marichette ». Pierre M. Gérin écrit à ce sujet :

Au grand scandale des esprits asservis à la grammaire, des puristes de tout poil qui reprochent à notre épistolière de donner un exemple coupable et de ruiner la réputation des Acadiens auprès des Français, voire des Anglais, au risque d'ailleurs de se faire mal comprendre même de ceux ou de celles qu'elle prétend

\footnotetext{
${ }^{3}$ Étant donné que j'étais en charge de ce séminaire, c'est moi qui ai sélectionné, après une lecture assidue, des passages à traduire. Il est nécessaire de souligner ici le fait que, bien que ce soit la professeure qui avait choisi les passages à traduire après une lecture assidue de la totalité des «Lettres », les étudiants n'ont dû respecter aucune contrainte imposée par l'enseignante : il ne s'agissait pas d'une pédagogie directive mais d'une vraie expérience de traduction.
} 
représenter, Marichette a donc choisi, par fidélité patriotique, d'écrire dans le parler des siens. (1982 : 120, nous soulignons.)

En effet, c'est ce "parler des siens », le parler traditionnel acadien, qui semble constituer un obstacle infranchissable dans le processus traduisant. Si l'on considère les parlers franco-acadiens comme une survivance en Amérique du Nord des formes linguistiques jadis utilisées en France métropolitaine, mais aujourd'hui tombées en désuétude, pour établir l'origine du parler acadien il faut de toute nécessité remonter au français des $\mathrm{XV}^{\mathrm{e}}$ et $\mathrm{XVI} \mathrm{e}^{\mathrm{e}}$ siècles. Les différences entre le français de cette époque et le français «de référence » contemporain sont nombreuses, et ceci est visible à tous les niveaux : lexical, morphologique, syntaxique, stylistique et phonétique. Ainsi, le premier problème qui apparaît lors de la lecture des «Lettres de Marichette » et des tentatives de les rendre en polonais s'avère le «mélange de formes» (Gérin 1982 : 123), «survivance de l'ancien et du moyen français » (Ibid.) à laquelle s'ajoutent les emprunts à des parlers divers. Pierre Gérin et Pierre M. Gérin caractérisent le langage de Marichette de manière suivante :

À la diversité d'origine du vocabulaire, la langue de Marichette ajoute un surprenant mélange de formes: les unes sont de simples survivances de l'ancien et du moyen français, d'autres semblent empruntées à des parlers aussi différents que le poitevin, le berrichon, le normand, voire le wallon ou le francoprovençal, d'autres enfin se trouvent encore bien vivantes aujourd'hui dans les classes peu instruites de la société française (Ibid. : 123).

Ce «mélange de formes » devient d'autant plus difficile à rendre dans la traduction vers le polonais que la langue polonaise ne dispose pas d'un nombre aussi élevé de variations dialectales et régionales. À cela s'ajoute l'absence des langues minoritaires en Pologne.

Le deuxième problème évoqué par les étudiants comme difficile à surmonter constitue l'inclusion de l'anglais dans l'original. En Acadie comme ailleurs au Canada français minoritaire, la rencontre des francophones avec l'Autre anglophone souvent d'origine immigrante crée des contextes multilingues dans lesquels une identité pluriculturelle ${ }^{4}$ est en train de se manifester et de se structurer. Autrement dit, dans le contexte des minorités francophones de l'exiguïté canadienne, la présence de la culture anglophone de plus en plus hybride et la recherche identitaire constante engendrent «la création de plusieurs identités chez le même individu, ainsi que l'appartenance de cet individu à plusieurs groupes sociaux : linguistiques, culturels ou ethniques » (Lafontant 2002 : 83). Il devient nécessaire alors de négocier entre les

\footnotetext{
${ }^{4}$ Nous comprenons le terme identité, d'après G. Marchildon (2001 : 27), en tant qu'identité sociale de l'individu résultant de son appartenance à un ou à plusieurs groupes et non en tant qu'identité exclusivement ethnique.
} 
différents pôles identitaires et de représenter les tensions et les doutes identitaires dans les textes littéraires, l'introduction des anglicismes y jouant un rôle important. Sherry Simon précise à ce propos que : « Les zones interlinguistiques [...] deviennent un lieu de création culturelle, qui exprime le caractère inachevé et transitoire des identités » $(1999: 40)^{5}$. Ainsi, les écrivains tentent de rendre compte du phénomène de rencontre des langues et du processus de construction identitaire dans une société multilingue. Pour y parvenir, les écrivains se servent à l'écrit comme à l'oral de $l^{\prime}$ hybridation linguistique ${ }^{6}$. L'inclusion de l'anglais devient alors une marque de la "pluralité identitaire » (Lüdi 2001 : 27) des personnages littéraires. Ceci semble aussi être une façon pour eux de suggérer la résistance des protagonistes à l'assimilation. Le recours aux emprunts à l'anglais peut être aussi lu comme une manière d'introduire une couleur locale et de réaffirmer certaines réalités culturelles francophones ${ }^{7}$. Ainsi, tout acte de communication produit dans le milieu francophone du Canada, qu'il soit d'ordre discursif ou littéraire, favorise la diversité culturelle et suppose la présence de l'Autre anglophone, perçue comme un ennemi acharné ou bien comme une identité supplémentaire et indispensable dans la création de l'identité commune franco-canadienne.

Ceci est d'autant plus pertinent en ce qui concerne le milieu francophone acadien du Nouveau-Brunswick, et pour maintes raisons; la plus importante résulte $\mathrm{du}$ fait que cette province est la seule province canadienne officiellement bilingue. Comme dans les autres provinces canadiennes, exception faite du Québec, les francophones au Nouveau-Brunswick constituent une minorité, quoiqu'une minorité tout à fait considérable (34\% de la population).

Une autre explication de la forte présence des anglicismes ${ }^{8}$ chez les Acadiens est l'aliénation de la population acadienne par rapport aux Québécois. Selon Paul Dubé, le changement encouru dans les relations avec le Québec a créé chez les communautés de l'exiguïté «un véritable traumatisme identitaire » (2001: 48),

\footnotetext{
${ }^{5}$ Il faut préciser ici que Sherry Simon, bien qu'elle se rende compte de ces tensions, met l'accent surtout sur le côté positif de la « création culturelle».

${ }^{6}$ Ce procédé peut en outre servir de rappel que le Canada est un pays multiethnique; l'écrivain souligne ainsi son appartenance à plusieurs codes linguistiques. Néanmoins, le paysage multiculturel et multilinguistique canadien nous donne des textes hybrides dont le transfert vers les cultures où le multilinguisme n'existe pas peut s'avérer difficile, comme c'est le cas en Pologne à l'heure actuelle.

${ }^{7}$ Bien que le multilinguisme se manifeste en Pologne dans les zones frontalières, les textes hybrides ne sont pas très présents dans la littérature polonaise, car le multilinguisme se manifeste à un degré beaucoup moins important qu'au Canada. On peut citer quelques exemples de l'inclusion du russe dans les textes polonais (Gustaw Herling-Grudziński, Inny świat ou Andrzej Pilipiuk, Weźmisz czarno kure), mais ce phénomène reste toujours marginal.

${ }^{8}$ Il faut expliquer ici que les étudiants participant au programme de traduction ne voyaient pas de différence entre les termes «l'inclusion de l'anglais » et « les anglicismes ». Pour eux, les deux notions renvoient au même phénomène: nous allons alors utiliser ces deux termes dans la suite de notre article.
} 
puisque l'effacement de l'ancienne dimension culturelle, liée à l'idée de la nation canadienne française, n'a pas engendré l'apparition d'une nouvelle forme politique d'identification. En plus, selon Dubé :

...la fragmentation relativement rapide de l'ancien espace français, dont le Québec s'était graduellement détaché tant sur le plan territorial qu'identitaire, entraîna la création de collectivités francophones distinctes, découpées selon les frontières des provinces et des régions du Canada. Les minorités d'expression française du Canada sont alors forcées de miser sur le paramètre institutionnel, c'est-à-dire sur leur insertion dans la réalité politique régionale. En somme, la rupture des liens avec la communauté québécoise les a obligées de délaisser les appareils idéologiques de la nation canadienne française au profit d'outils mieux adaptés à leur nouvelle réalité provinciale, en favorisant de cette manière la diversité culturelle. (2001 : 50)

La difficulté à se forger une identité "distincte» a débouché sur une indécision identitaire et sur la peur de l'ethnicisation à laquelle on essaye de reléguer les minorités de l'exiguïté.

Inclure l'anglais dans les « Lettres » devient ainsi l'un des problèmes majeurs de la traduction, puisqu'il est impossible de faire alterner la langue anglaise et polonaise d'une façon naturelle et compréhensible pour le lecteur polonais.

Les deux problèmes dont nous venons de discuter se rencontrent dans le troisième obstacle à la traduction des «Lettres de Marichette »: le vocabulaire hétérogène et la structure des phrases qui ne correspond pas au français de la métropole, considéré par les étudiants comme normatif. Il est surtout question des survivances grammaticales et lexicales de l'ancien et du moyen français, ainsi que des variantes dialectales déjà mentionnées. Ces trois problèmes principaux sont ceux que les étudiants ont remarqués immédiatement, lors de la première lecture des «Lettres ». Situés au niveau linguistique et sociolinguistique, ils résultent de la distance qui sépare la langue et la culture polonaises de la langue et la culture francoacadiennes. Les problèmes de traduction du français acadien au polonais, restreints jusqu'à présent à la question linguistique, se présentent de la manière suivante :

a) absence en polonais d'un nombre aussi élevé de variations dialectales et régionales; l'absence des langues minoritaires en Pologne rend impossible la recherche de l'équivalent exact,

b) impossibilité de faire alterner les deux langues (anglais et polonais) d'une manière naturelle, comme c'était le cas chez Marichette,

c) difficulté de rendre les survivances de l'ancien et du moyen français. 
$\mathrm{Vu}$ ces problèmes de traduction, il devient évident que le parler acadien des «Lettres de Marichette» ne peut être transféré dans la langue polonaise que partiellement; il s'agira donc d'une stratégie d'adaptation et non d'équivalence (Hurtado-Albir 1990 : 35-47).

\section{Stratégies de traduction employées par les étudiants}

Avant d'entamer notre analyse il faudrait souligner encore une fois que nous n'avons pas dirigé la prise de décision des étudiants. Au contraire, nous avons encouragé la créativité linguistique et l'imagination des étudiants afin de leur permettre de découvrir les stratégies de traduction propices à rendre l'original en polonais. Toutefois, il s'est avéré que leur réaction initiale était surtout affective, car en réalité la langue polonaise dispose des moyens linguistiques qui permettent le transfert des «Lettres de Marichette » dans la culture polonaise. L'analyse du résultat final nous a permis d'établir une classification des principales stratégies utilisées pendant le processus traduisant.

1) Emploi du langage populaire contemporain (pour rendre le parler acadien):

a) Lettre du jeudi 28 février 1895: "Billie chi a venu me passer son poing sous l'nez » (p. 115) - traduction polonaise : «Nawet Billie mi dupe zawraca» (p. 7), (Même Billie me fait chier)

b) Lettre du jeudi 18 mars 1897: "L'autre dimanche j'étions à l'église, et y avait du. monde à faire trember! » (p. 158) - traduction polonaise : «Poszłam zeszłej niedzieli do kościoła, w cholere wiary tam było » (p. 11) (L'autre dimanche je suis allée à l'église, et il y avait du monde à en vomir!)

Les fragments soulignés signalent une certaine consternation que les étudiants ont manifestée face aux exemples du parler de Marichette, avec ses expressions figées et son vocabulaire spécifique, et qu'ils ont tenté de rendre à l'aide des constructions provenant du langage populaire utilisé de nos jours en Pologne. Il est vrai que ce langage dénote plutôt l'appartenance à des classes inférieures de la société polonaise, classes plutôt rurales et sous scolarisées (vu sous cet angle, il pourrait effectivement constituer le moyen approprié pour rendre en polonais le parler de Marichette), néanmoins les structures linguistiques polonaises choisies par les étudiants sont associées en Pologne principalement au langage des jeunes. En plus, ces structures se placent généralement à un niveau plus élevé de grossièreté par rapport aux constructions de l'original. Il faudrait aussi souligner l'emploi d'un lexème appartenant au parler populaire de la région de Poznań en particulier et de la Grande Pologne en général : le mot wiara ${ }^{9}$ (foule, plein de monde) employé dans le deuxième exemple confirme effectivement ce que nous venons de dire plus haut, à

\footnotetext{
${ }^{9}$ Ce mot est décliné dans l'exemple analysé, d'où la différence dans l'écriture (wiara-wiary).
} 
savoir que la langue polonaise dispose des moyens linguistiques assez diversifiés au niveau régional pour permettre la traduction du parler de Marichette.

2) Emploi des formes dialectales et régionales:

a) au niveau phonétique afin de remplacer le parler acadien de Marichette, p. ex., les formes: chi, chisque, matagne, les traducteurs ont décidé d'employer les variantes dialectales polonaises, telles que : (i) changement $\underline{\mathrm{o}-\mathrm{u}}$ : $k$ toś $^{10}-k t u s$ s (quelqu'un); (ii) changement $\underline{\text { a-o }}$ : plugastwo-plugostwo (cochonnerie, insulte), zawraca-zawroca (il retourne); (iii) changement $\underline{\mathrm{e}-\mathrm{y}}$ : te $\dot{z}-t y \dot{z}$ (aussi), ten-tyn (celui-ci); (iv) disparition des voyelles nasales terminales et à l'intérieur du mot: przeszkadzaja-przeszkadzajom (ils dérangent), przeklęty-przeklenty (sacré), moge-mogem (je peux).

b) au niveau morphologique et syntaxique, l'emploi des formes dialectales concerne ici :

(i) la place du verbe: à la fin de la phrase pour rendre la valeur régionale ${ }^{11}$,

(ii) changement des terminaisons verbales; ce procédé sert à exprimer les constructions utilisées par Marichette, p. ex., j'avons, il a venu, etc. : poszlim-poszliśmy (nous sommes allés); le raccourcissement des terminaisons verbales indique en polonais l'appartenance du locuteur à la classe inférieure, voire son origine rurale;

(iii) terminaison verbale avant le radical: żeście opublikowaliopublikowaliście (vous avez publié), żem poszła-poszłam (je suis allée). Ce procédé linguistique diffère considérablement du précédent, car l'emploi de la terminaison verbale avant le radical est très courant dans la langue polonaise contemporaine et ne constitue pas l'exclusivité du langage populaire. Au contraire, cette construction linguistique trouve sa place dans le polonais standard. C'est l'utilisation en abondance de cette structure (dans chaque proposition ou presque) qui permet au traducteur d'obtenir la valeur dialectale et archaïque.

c) au niveau lexical, l'emploi des variantes dialectales de la région de Silésie: fagas (type), fanzolić (raconter des bêtises) et de la région de Poznań : banapociag (train), wiara-ludzie (gens). Les étudiants ont choisi d'utiliser deux dialectes pour traduire le parler acadien: le dialecte silésien et celui de la Grande Pologne, la région où ils habitent. La Pologne ne possédant pas de langues minoritaires ou de dialectes dont la pertinence serait ressentie dans les médias les étudiants ont misé plutôt sur les constructions dialectales qui sont

\footnotetext{
${ }^{10}$ Dans tous les exemples présentés dans ce paragraphe la première forme en italique est toujours la forme en polonais standard, la deuxième étant une structure régionale ou bien dialectale.

${ }^{11}$ Dans la langue polonaise le placement du verbe à la fin d'une proposition complexe indique toujours une valeur régionale, voire archaïque, d'où l'utilisation très fréquente de cette méthode par les étudiants dans la traduction des « Lettres ».
} 
entrées dans le polonais standard ou bien sur celles qu'ils connaissent personnellement.

\section{3) Archaïsation du texte traduit}

Se servant très peu des variantes dialectales ou régionales du polonais, les étudiants ont plutôt choisi d'archaïser le texte traduit afin de rendre "les survivances de l'ancien et du moyen français » (Gérin 1982 : 123) présentes dans le parler acadien de Marichette. Deux stratégies d'archaïsation ont été employées :

a) au niveau syntaxique: la même chose que pour les variantes régionales, les dialectes ayant gardé des formes anciennes de la langue polonaise

b) au niveau lexical :

i) connecteurs, adverbes: jeno, ino-tylko (uniquement), pewnikiem-pewnie (sûrement), kogóż kogo (qui), wpodle-obok (à côté)

ii) substantifs : niewiasta-kobieta (femme), łgarstwo-kłamstwo (mensonge), gawiedź-ludzie (gens)

iii) constructions phraséologiques: zachodzić w głowę-zastanawiać się (se casser la tête), kogóż ujrzeć mi przyszło-kogo zobaczyłam (qui viens-je de voir?)

L'emploi des constructions ou des lexèmes dont l'origine remonte au $\mathrm{XIV}^{\mathrm{e}}$ ou au $\mathrm{XV}^{\mathrm{e}}$ siècle a permis de montrer au lectorat polonais potentiel la richesse et la spécificité du langage utilisé par l'épistolière. Mais il est nécessaire d'ajouter que les traits dénotant le parler de Marichette en tant que français acadien disparaissent inévitablement dans la traduction. Tout de même, cette perte semble être minime, car grâce aux stratégies de traduction employées, le lecteur polonais moyen est capable de reconnaître que Marichette parle différemment par rapport aux règles du polonais standard, qu'elle utilise une variante dialectale de la langue et qu'elle est d'origine rurale. Si le récepteur peut facilement situer le personnage dans un milieu rural isolé et peu scolarisé, il aura probablement la difficulté à le situer dans le temps : la traduction ne réussit ainsi pas à introduire une distance historique remontant au XIXe siècle.

\section{4) Élimination de la langue anglaise}

$\mathrm{Du}$ point de vue méthodologique, cette partie est problématique. Reproduire le phénomène de langues en contact, dont une majoritaire et dominante et l'autre minoritaire et dominée, s'avère très difficile, étant donnée l'histoire coloniale du Canada qui n'est pas partagée avec la Pologne. Dans le contexte polonais, seule la présence de l'allemand et du russe dans certaines zones frontalières pourrait signaler une situation semblable. L'emprunt à l'anglais en polonais dans la situation actuelle ne peut être comparé au contexte linguistique acadien. Par conséquent, l'inclusion de l'anglais dans le texte français des «Lettres » a été jugée par les étudiants comme un problème majeur de traduction. Employer l'anglais dans le texte polonais pourrait non seulement provoquer des malentendus au niveau de la compréhension (la 
connaissance de l'anglais par les Polonais n'étant pas généralisée), mais aussi nuire à la réception $\mathrm{du}$ texte traduit dans sa totalité. Les étudiants ont finalement décidé d'effacer complètement la langue anglaise de la traduction ou bien de laisser uniquement les structures ou les mots dont ils ont jugé la compréhension facile de la part du lecteur polonais.

a) élimination complète :

Lettre du jeudi 3 octobre 1895: "il est smart on $m^{\prime} d i t »(p .118)$ - « gadaja, co on bystry jest » (p. 7)

b) élimination partielle :

Lettre du jeudi 4 juin 1896 : «good for Marichette, write some more » (p. 144) " good for Marichette, napisz coś jeszcze » (p. 9) (écris quelque chose d'autre).

\section{5) Explicitation des éléments culturels}

L'orientation sociale à l'intérieur d'un même espace culturel s'effectue à l'aide de symboles culturels spécifiques, lesquels sont acquis par l'individu au cours de sa vie. Cette capacité de reconnaissance et d'interprétation, cette compétence de compréhension permet de se situer et de s'orienter dans sa culture, et rend l'individu capable d'agir et de communiquer socialement, en partageant avec les membres de la même communauté linguistique et culturelle des évidences, des non-dits qu'il a intégrés et qu'il suppose assimilés de la même façon par les autres. De sorte que la base réelle d'une culture soit avant tout la connaissance essentiellement implicite du réseau de connotations, des significations, des valeurs affectives et sociales rattachées aux signes observables. Et c'est justement cette connaissance-là qui détermine les choix traductologiques. Lors d'un acte de communication (qui s'effectue ici par le biais de la littérature), ce savoir partagé s'actualise autant dans le discours que dans le comportement de chacun des partenaires. Les associations, les analogies, les catégorisations stéréotypées et d'autres opérations cognitives semblables constituent une part importante du processus de compréhension. Lorsqu'il s'agit d'un échange culturel qui s'effectue entre deux cultures mineures (ici, la culture acadienne et polonaise) qui n'ont aucune histoire de contact sinon des contacts isolés, la couche culturelle joue un rôle primordial dans la compréhension du message traduit. Les étudiants ont décidé donc dans la majorité des cas d'expliciter le réseau connotatif présent dans le texte afin que le message implicite transmis dans l'original soit, au moins partiellement, saisi par le lecteur polonais.

a) explicitation dans le texte

Lettre du jeudi 18 mars 1897: "J'crois qu'ceux là sont des torys » (p. 142) «Pewnikiem sam $z$ torysów, tych przeklentych konserwatystów» (ces foutus conservateurs), (p. 12).

L'ajout d'un adjectif qualificatif sous forme d'épithète a pour but de montrer au lecteur polonais, dont le bagage cognitif ne permet pas de saisir la couche 
connotative du mot torys, la signification implicite que Marichette associe à ce lexème.

b) explicitation sous forme des notes du traducteur. Les annotations contiennent les explications concernant l'Évangéline, ainsi que d'autres noms propres.

Les étudiants se servent très souvent des annotations pour expliquer au lecteur potentiel la signification des mots ou des structures enracinés culturellement dans la communauté acadienne. Nous ne développons pas davantage cette problématique fort intéressante en soi puisque nous avons consciemment choisi les passages des «Lettres de Marichette » où la couche connotée culturellement n'était pas un élément dominant du sens.

\section{Conclusion}

L'existence d'un grand nombre des langues présuppose l'existence des traductions. Pour que les gens puissent communiquer sans obstacles, nous avons besoin de quelqu'un qui puisse faciliter cette communication. Les personnes qui traversaient et traversent toujours les frontières linguistiques, ce sont bien évidemment les traducteurs qui, depuis toujours, ont dû «transmettre un même sens avec des moyens linguistiques différents » (Hurtado-Albir 1990: 90). La traduction des romans à caractère plurilingue, où la rencontre avec l'Autre devient en même temps le choix individuel d'une configuration identitaire, est encore plus difficile justement à cause de cette alternance codique (code-mixing) et culturelle que nous avons évoquée dans les paragraphes précédents. De plus, elle devient problématique lorsque le choix langagier implique le changement affectif ou implicite du discours littéraire.

Notre expérience de traduction qui a eu lieu dans le cadre de la formation universitaire est une intervention visant à mieux faire connaître le Canada francophone en Pologne. La collaboration entre des traductologues et des étudiants polonais en philologie romane favorisera l'étude des pratiques retenues par le traducteur polonais de la littérature canadienne-française. Cela permettrait non seulement de mieux connaître en Pologne les nouveaux courants de la traductologie élaborés par des traductologues canadiens, mais aussi dans un cadre plus général, et en particulier politique, de mieux cerner l'intérêt de la Pologne envers le Canada. Cette expérience a aussi pour but de faire connaître aux étudiants polonais la diversité de la francophonie ainsi que le phénomène du plurlinguisme au sein de cette francophonie, vu qu'ils ont parfois tendance à oublier que la langue française dite «standard " n'est pas le seul moyen d'exprimer son origine francophone. Par ailleurs, cette expérience a permis aux étudiants de ressentir un certain contact interculturel avec l'autre francophone minoritaire du Canada. En plus, nous considérons que ce programme de traduction est très important pour l'institution littéraire en Pologne puisque la présence des textes hybrides sur la scène littéraire 
polonaise s'inscrit dans un courant plus large de métissage culturel, courant jusquelà peu présent en Pologne. L'espace culturel et littéraire contemporain est traversé par une multitude de langages, de réalités, de textes, de cultures et de discours; ses articulations et ses manifestations ne peuvent plus se passer de notions telles que l'hétérogénéité, la pluralité, l'hybridité, la diversité, la fragmentation, etc. Dans une telle constellation de valeurs, où le paradigme de la diversité domine, tout discours ou texte culturel unitaire et homogène ne véhicule pas de symbolique capable de susciter une interaction avec le récepteur.

L'acceptation de la différence constitue le pivot d'un véritable interculturalisme. Dans ce contexte, la traduction des romans acadiens ne peut être possible qu'à condition d'élargir la notion de la culture francophone et de prendre pour acquis le fait que « la culture francophone sera désormais le lieu où se situent la multiplicité, la différence, l'altérité... » (Dubé 2001 : 54). Lors de la rencontre de deux petites cultures par le biais de la traduction, l'altérité et la diversité se manifestent comme les aspects les plus importants à transmettre dans la culture réceptrice. La rencontre d'une alternative francophone véhiculée dans la littérature acadienne et des traducteurs polonais motivés à connaître cette expression d'altérité plurielle en fait foi. 


\section{BIBLIOGRAPHIE}

\section{Corpus}

GÉRIN, Pierre et GÉRIN, Pierre Marie (1982), Marichette. Lettres acadiennes 1895-1898. Édition commentée, Sherbrooke, Éditions Naaman.

\section{Documentation théorique}

DEMORGON, J (2003), L'histoire interculturelle des sociétés, Paris, Anthropos.

DUBÉ, Paul (2001), «Francophonies exiguës et mondialisation. Métissages et dialectique identitaire », dans Actes du colloque Francophonie au pluriel, organisé par Année Internationale Francophone, Paris, PUF, 47-55.

GIGUÈRE, Alain (2006), « Le Canada fout le camp », L'Actualité, février, 11-13. HURTADO-ALBIR, Amparo (1990), La notion de fidélité en traduction, Paris, Didier Éruditions.

LAFONTANT, Jean (2002), «Langue et identité culturelle : points de vue des jeunes francophones du Manitoba », Francophonies d'Amérique 14, 81-88.

LÜDI, Georges (2001), «Le mélange de langue comme moyen stylistique et/ou marqueur identitaire dans le discours littéraire », Creliana, hors série 1, 13-31.

MARCHILDON, Gilles (2001), «Être 'vrai' Franco-Manitobain », La Liberté 87/41, 2631.

SIMON, Sherry (1999), Hybridité culturelle, Montréal, Île de la Tortue. 